\title{
Re-Evaluation of RF Electromagnetic Communication in Underwater Sensor Networks
}

Xianhui Che, lan Wells, Gordon Dickers, Paul Kear, and Xiaochun Gong, Swansea Metropolitan University

\begin{abstract}
Most underwater wireless networks use acoustic waves as the transmission medium nowadays, but the chances of getting much more out of acoustic modems are quite remote. Optical links are impractical for many underwater applications. Given modern operational requirements and digital communications technology, the time is now ripe for re-evaluating the role of electromagnetic signals in underwater environments. The research presented in this article is motivated by the limitations of current and established wireless underwater techniques, as well as the potential that electromagnetic waves can offer to underwater applications. A case study is presented that uses electromagnetic technology in a small-scale underwater wireless sensor network. The results demonstrate the likely effectiveness of the designated network.
\end{abstract}

\section{INTRODUCTION}

Underwater electromagnetic (EM) communications were investigated with fervent interest in the last century up until the 1970s. Because its range is restricted by fundamental attenuation and noise factors that must be considered as unchangeable environmental elements, significant breakthroughs were not to be expected in submarine radio communication [1]; hence, radio communication, although it has notable merits in the terrestrial wireless network field, has had very few practical underwater applications to date.

In the present digital era, benefits of shortrange and high-bandwidth communications systems have become familiar to users. Meanwhile the oil industry, military, and environmental operations are demanding reliable, connectorless, and short-range data link applications; consequently, it is time to re-evaluate EM capabilities in the underwater environment. EM signaling, coupled with digital technology and signal compression techniques, has many advantages that make it suitable for niche underwater applications.

Recent activities in monitoring aqueous environments have led to extensive research on underwater wireless sensor networks (UWSNs), most of which have used acoustic waves as the physical transmission medium. Acoustic transmissions outperform EM for vertical range and represent the best engineering solution in most long-distance applications. However, refraction in deep water, thermal gradients, and reflections in shallow water pose operational limits and call for alternatives. Because EM signaling uses a different transmission mechanism than acoustic, it can extend the arena of application, and there is little overlap in operational conditions for the two techniques. Acoustic and EM techniques can be viewed as complementary technologies. Another option for underwater wireless communication is optical wave technology, which has also recently drawn increasing attention from academia and researchers. Compared to acoustic and optical wave technologies, radio frequency (RF) EM technology offers great potential for underwater sensor communications that are worth addressing.

In this article the next section gives an overview of the three major technologies acoustic, RF, and optical - and compares their benefits and limitations. The details of underwater RF-EM performance are then discussed, along with the highlights of merits and capabilities that make it suitable for underwater applications. We then describe a case study that attempts to use RF-EM communication in a UWSN for coastal monitoring purposes. We then analyze the prospects of UWSN development with RFEM communication and summarize the challenges of its design and implementation. The final section concludes the article.

\section{OVERVIEW OF UNDERWATER WIRELESS COMMUNICATION TECHNOLOGIES}

Extensive research and development has been carried out in underwater acoustic networks [2-4]. Acoustics is a proven technology for underwater sensor applications which offers long transmission ranges of up to $20 \mathrm{~km} \mathrm{[5],} \mathrm{although}$ certain challenges and limitations have also been revealed $[6,7]$. Acoustic waves yield poor performance in shallow water where transmission can 
- Crosses air/water/seabed boundaries easily

- Prefers shallow water

RF

- Unaffected by turbidity, salinity, and pressure gradients

- Works in non-line-of-sight; unaffected by sediments and aeration

- Immune to acoustic noise

- High bandwidths (up to $100 \mathrm{Mb} / \mathrm{s}$ ) at very close range
- Susceptible to EMI

- Limited range through water

- Strong reflections and attenuation when transmitting through water/air boundary

- Poor performance in shallow water

- Adversely affected by turbidity, ambient noise, salinity, and pressure gradients

- Limited bandwidth (0 b/s to $20 \mathrm{~kb} / \mathrm{s})$

- Impact on marine life

- Does not cross water/air boundary easily

- Susceptible to turbidity, particles, and marine fouling

- Needs line-of-sight

- Requires tight alignment of nodes

- Very short range

Table 1. Comparison of underwater wireless communication technologies.

be affected by turbidity, ambient noise, salinity, and pressure gradients; in addition, acoustic technology can have an adverse impact on marine life [8].

The very high capacity of optical wave technology has recently stimulated several attempts at research on underwater optical communications, of which the latest include [9-12]. However, optical waves only deliver good performance in very clear water and require tight alignment of nodes. The requirement for line of sight when using optical communications has imposed a significant constraint on its underwater application, although efforts have been made in [11] to try to overcome such a limitation.

Even though RF-EM can suffer from limited transmission range and electromagnetic interference (EMI), it also has some valuable features that can enable flexible deployment of UWSNs in coastal regions. Research on underwater EM communication is currently ongoing, with examples such as $[13,14]$. Table 1 outlines the three major underwater communication technologies in terms of benefits, limitations, and requirements. Compared to acoustic and optical technologies, RF-EM has some distinct advantages that make it suitable for underwater environments.

First, both acoustic and optical waves cannot perform smooth transitions through the air/water interface. EM waves can cross water-to-air or water-to-earth boundaries easily following the path of least resistance. In this way, both air and seabed paths will act to extend the transmission range. Should this feature be fully taken advantage of, it can make a significant contribution to network design and implementation. More details on this aspect are discussed in the following sections. Second, EM transmissions are tolerant to turbulence caused by tidal waves or human activities, as opposed to acoustic and optical waves, which are not. This feature is certainly beneficial to the system performance in random and unpredictable underwater environments. Third, EM waves can work in dirty water conditions, while optical waves are susceptible to particles and marine fouling. This gives EM an advantage when working in a water column with a high level of sediment and aeration. Fourth, unlike acoustic technology, EM radiation is immune to acoustic noise, and it has no known effects on marine life.

\section{UNDERWATER RF-EM PERFORMANCE}

\section{Key PARAMETERS}

EM propagation through water is very different from propagation through air because of the high permittivity and electrical conductivity of water. Propagating waves continually cycle energy between electric and magnetic fields; hence, high conduction leads to strong attenuation. Plane wave attenuation also increases rapidly with frequency.

Table 2 shows the variation of key parameters vs. frequency, which is based on the equations below. $\lambda$ is the wavelength, $f$ is the frequency, $\sigma$ is the conductivity, $v$ is the propagation velocity, and $\delta_{\text {skin }}$ is the skin depth. The derivations of these equations are detailed in Appendix A. Figures in this table have been acquired using representative conductivity value $(\sigma=0.01 \mathrm{~S} / \mathrm{m})$ which is typical for fresh water. As for the sea water conductivity, $\sigma$ is set to 3.2 , $4.2,4.3,4.3,4.3$, and $5.4 \mathrm{~S} / \mathrm{m}$ for the respective frequencies listed in the table.

$$
\begin{aligned}
& \lambda=1 / \sqrt{f \sigma \times 10^{-7}} \\
& v=\sqrt{f \times 10^{7} / \sigma} . \\
& \delta_{\text {skin }}=1 /\left(2 \pi \sqrt{f \sigma \times 10^{-7}}\right)
\end{aligned}
$$

For comparison purposes, acoustic propagation velocity is also presented as a frequency-invariant $1500 \mathrm{~m} / \mathrm{s}$. Above $10 \mathrm{kHz}$ EM signals propagate 


\begin{tabular}{|c|c|c|c|c|c|c|c|}
\hline & & \multicolumn{6}{|c|}{ Frequencies (Hz) } \\
\hline & & 100 & 1000 & 10,000 & 100,000 & 1 million & 10 million \\
\hline \multirow{4}{*}{ Propagation velocity $(\mathrm{m} / \mathrm{s})$} & Sea water & $1.77 \times 10^{4}$ & $4.88 \times 10^{4}$ & $1.52 \times 10^{5}$ & $4.82 \times 10^{5}$ & $1.52 \times 10^{6}$ & $4.30 \times 10^{6}$ \\
\hline & Fresh water & $3.16 \times 10^{5}$ & $1.00 \times 10^{6}$ & $3.16 \times 10^{6}$ & $1.00 \times 10^{7}$ & $3.16 \times 10^{7}$ & $1.00 \times 10^{8}$ \\
\hline & Free space & $3.00 \times 10^{8}$ & $3.00 \times 10^{8}$ & $3.00 \times 10^{8}$ & $3.00 \times 10^{8}$ & $3.00 \times 10^{8}$ & $3.00 \times 10^{8}$ \\
\hline & Acoustic & $1.50 \times 10^{3}$ & $1.50 \times 10^{3}$ & $1.50 \times 10^{3}$ & $1.50 \times 10^{3}$ & $1.50 \times 10^{3}$ & $1.50 \times 10^{3}$ \\
\hline \multirow{2}{*}{ Wavelength (m) } & Sea water & $1.76 \times 10^{2}$ & $4.88 \times 10^{1}$ & $1.52 \times 10^{1}$ & $4.82 \times 10^{0}$ & $1.52 \times 10^{0}$ & $4.30 \times 10^{-1}$ \\
\hline & Free space & $3.00 \times 10^{6}$ & $3.00 \times 10^{5}$ & $3.00 \times 10^{4}$ & $3.00 \times 10^{3}$ & $3.00 \times 10^{2}$ & $3.00 \times 10^{1}$ \\
\hline \multirow{2}{*}{$\begin{array}{l}\text { Propagation distance }(\mathrm{m}) \\
\text { for } 100 \mathrm{~dB} \text { attenuation }\end{array}$} & Sea water & $3.23 \times 10^{2}$ & $8.92 \times 10^{1}$ & $2.79 \times 10^{1}$ & $8.81 \times 10^{0}$ & $2.79 \times 10^{0}$ & $7.87 \times 10^{-1}$ \\
\hline & Fresh water & $5.78 \times 10^{3}$ & $1.83 \times 10^{3}$ & $5.78 \times 10^{2}$ & $1.83 \times 10^{2}$ & $5.78 \times 10^{1}$ & $1.83 \times 10^{1}$ \\
\hline
\end{tabular}

Table 2. EM performance underwater.

more than 100 times faster than acoustic. This has important advantages for command latency and networking protocols where many signals have to be exchanged. The second part of Table 2 shows the impact on wavelength by increasing frequency, and highlights the degree to which a wavelength is shortened under water: for instance, at $100 \mathrm{~Hz}$ the free space wavelength is $3000 \mathrm{~km}$ while in sea water it is only $176 \mathrm{~m}$. This effect has important implications for sensing and navigation applications where the frequency required for a specified dimensional resolution is much lower than in air. The third part of the table shows a simple quantitative illustration of range with the impact of increasing frequency. Distances have been calculated for $100 \mathrm{~dB}$ attenuation of a propagating plane wave using the equation below, where $e$ is the Euler's number $(e \approx 2.72)$.

$$
\frac{20 \log _{10}(1 / e)}{\delta_{\text {skin }}}=\frac{100 \mathrm{~dB}}{\text { distance }}
$$

It is interesting to consider the reversal of roles between acoustic and EM technologies when passing from water to air. In air acoustic signals are highly attenuated, so hundreds of people can hold separate conversations in a crowded conference hall. Radio waves have low attenuation in air, so communications must be separated in frequency through careful management of the spectrum. In water the two roles reverse themselves, and it is easy to see the potential benefits of high EM spatial attenuation in a multi-user environment, which enables localized communications.

\section{Multipath Propagation}

An important consideration for underwater RFEM performance is the effect of the water-to-air interface. Propagation loss and the refraction angle are such that an EM signal crosses the water-to-air boundary and appears to radiate from a patch of water directly above the transmitter. The large refraction angle produced by the high permittivity launches a signal almost parallel with the water surface. This effect aids communication from a submerged station to the land and between shallow submerged stations without the need for surface repeater buoys. A similar effect can also be produced at the seabed. Since the conductivity of the seabed is much lower than water, it can provide an alternative low-loss, low-noise, covert communications path.

Figure 1 illustrates the multipath propagation of EM waves underwater. In many deployments the single propagation path with the least resistance will be dominant. Should the air path or seabed path be chosen as the dominant data path, relatively longer transmission range can be achieved compared to the water path. Hence, the multipath propagation of EM waves can be advantageous for signal transmission in shallow water conditions.

The third part of Table 2 shows attenuation through the water path; but for communication between shallow submerged stations, most of the signals are carried by the air path, so the contents of Table 2 should not be interpreted using horizontal range measure alone. For example, if two stations are $1 \mathrm{~km}$ apart and $2 \mathrm{~m}$ below the surface, attenuation will be significantly less than anticipated from the $1 \mathrm{~km}$ through water loss. In ,comparison acoustic signals cannot cross the water-to-air boundary, so $1 \mathrm{~km}$ through water loss would apply.

\section{ANTENNAS}

Magnetic coupled loop antennas are the most compact practical solution for duplex submerged systems. Loop antennas are directional in nature, 


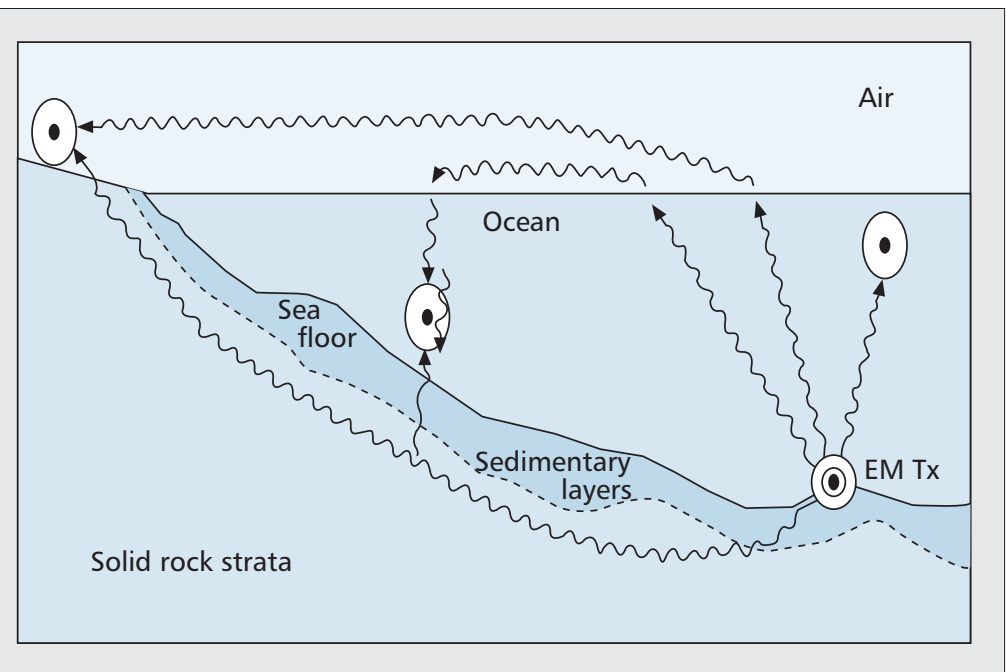

Figure 1. RF multi-path propagation underwater.
Short-range navigation systems can take advantage of the signal magnitude gradient seen in EM propagation. For beacon applications, sonar systems must use phase information to sense wave front direction, which suffers from multipath effects and pressure gradients. Navigation systems based on EM signals can measure increased signal strength as a direct response to movement toward a beacon, which enables a simple and robust control loop. Distributed cables can be designed to radiate an EM signal along their length. This type of distributed transducer has no equivalent in the acoustic domain. Some of these applications are currently being developed. For example, Wireless Fibre Systems (WFS) has demonstrated the latest results of using such technology in underwater navigation [18].

EM bandwidth only exceeds the counterpart acoustic systems at very short range. Most shortrange applications tend to be at depth and these systems have the advantage of effective shielding from environmental noise by the water column. Similarly, long-range applications operating from the surface to the seabed will experience an asymmetric noise environment as the shielding effect of the ocean lowers the noise experienced at the sea bed compared to that at the surface.

\section{CASE STUDY} ems can be designed using relatively compact loops, for instance, a small area loop antenna has been demonstrated in [15].

Another option is to use an electric dipole antenna for lateral electromagnetic waves [16]. Research in [13] has implemented a system using a crossed uninsulated terminated dipole with a diameter of $2 \mathrm{~cm}$ and $1 \mathrm{~m}$ in length, which makes careful reciprocal orientation unnecessary, thus simplifying deployment.

\section{Benefits AND Applications}

The benefits of EM signaling when applied to sensing, communications, and navigation are listed in Table 3, which indicate the great potential of RF-EM for underwater applications. In most applications it is the unique propagation mechanism that delivers niche advantages that can complement the use of existing underwater systems.

Table 4 summarizes realizable ranges and example bit rates for compact underwater EM communication systems. Based on the figures from Table 2, propagation velocity at higher frequencies changes less dramatically than at lower frequencies. Due to the nonlinear relationship between velocity and frequency, it is necessary to reduce bandwidth at low-range frequencies in order to minimize the effect of rapid velocity changes throughout the band. All the example scenarios given in Table 4 use 20 percent of the frequency value as the bandwidth, so relatively higher frequency carriers offer broader-band communication, and vice versa. The example data rates have been obtained using a binary phase shift keying (BPSK) modulation scheme where the bit rate is half the bandwidth [17]. A variety of practical applications can be implemented using underwater EM in sensing, communications, and navigation. Many of these applications are specifically desirable for autonomous underwater vehicles (AUVs).
In order to gather data and consequently quantify the effects of coastal erosion beneath the sea surface, the Automated Sensing Technology for Coastal Monitoring (ASTEC) project aims to create the world's first automated system for monitoring and forecasting for this purpose. In environmental science, coastal erosion effects can be indicated by the depth of closure (DoC), which is defined as the depth seaward where neither change in bottom elevation nor net sediment exchange between nearshore and offshore can occur significantly [19]; the contour of such closure is approximately parallel to the coastline in most cases. The primary role of sensors in the UWSN of this project will be to either directly or indirectly measure the movement of sea bed sediments so as to monitor the DoC.

\section{System ANd Network Design}

Based on the discussion in the previous section, each sensor is equipped with a magnetic coupled loop antenna. All the nodes in the network are battery driven and fixed in static underwater locations by buoys and ballasts. Figure 2 shows the block diagram of an end sensor node, an intermediate sensor node, and the sink, as well as the multipath propagation between the end sensor and the intermediate sensor.

A radio modem called Seatext [20] produced by WFS is employed to offer high-tolerance communications through water, air, and ground. As the modem is locked to a single frequency, the transceiver needs to be implemented in a half-duplex arrangement. As for the communication systems, experiments and measurements have been carried out to determine the following settings [21]. Very low frequency (VLF) $3 \mathrm{kHz}$ is used, and the data rate is set to $100 \mathrm{~b} / \mathrm{s}$. A $40 \mathrm{~m}$ 
Features

Crosses water to air boundary

Multipath less of an issue

Frequency-agile capability

Covert, localized communications

Performance High joules per bit efficiency

Potential for high data rates over small distances

High propagation speed

Unaffected by pressure gradients

Immune to acoustic noise

Reliability

Implementation

Unaffected by low visibility

Immune to aerated water

No need for surface repeater

Distributed transducers

Compact, portable units

No effects on marine animals
Details

Long-range horizontal communication using air path, water-to-air, or land

Can be advantageous in shallow water conditions

No mechanical tuned parts as in an acoustic system

Using high-frequency carrier for high attenuation; close spatial frequency reuse

For short-range and high-bandwidth applications, high bit rate results in efficient system in terms of joules per bit, extending deployment times for battery operated equipment

Use of $\mathrm{MHz}$ carrier; does not require precise navigation for hard docking of connector-based systems; improved reliability vs. connectors; avoids marine fouling, particulates, and alignment issues seen in laser-based systems

Low Doppler shift, low propagation delay especially important for networking protocols requiring multiple exchanges of information for handshake and error checking

Allows horizontal propagation

Operation unaffected by engine noise of heavy work, breaking waves, etc.

Sediment disturbed at the sea bed has no operational effect, while laser systems fail to operate

Operation in surf zone, communication at speed through cavitating propeller wash

Crosses water-to-air boundary for a long range without a surface repeater

Radiating cables can deliver unique navigation and communications functions

Small-to-medium antennas deliver acceptable performance

Effect of acoustic signals on marine mammals is becoming an issue

Table 3. Summary of advantages of underwater RF-EM technology.

\begin{tabular}{lllll} 
Range & $<10 \mathrm{~m}$ & $50 \mathrm{~m}$ & $200 \mathrm{~m}$ & $>1 \mathrm{~km}$ \\
\hline Sea water & $>8 \mathrm{~kb} / \mathrm{s}$ & $300 \mathrm{~b} / \mathrm{s}$ & $25 \mathrm{~b} / \mathrm{s}$ & $<1 \mathrm{~b} / \mathrm{s}$ \\
Fresh water & $>3 \mathrm{Mb} / \mathrm{s}$ & $150 \mathrm{~kb} / \mathrm{s}$ & $9 \mathrm{~kb} / \mathrm{s}$ & $<350 \mathrm{~b} / \mathrm{s}$ \\
\hline Applications & $\begin{array}{l}\text { AUV docking; } \\
\text { diver's personal network }\end{array}$ & $\begin{array}{l}\text { Networks; } \\
\text { diver conversation }\end{array}$ & $\begin{array}{l}\text { AUV control; } \\
\text { networking; } \\
\text { diver conversation }\end{array}$ & $\begin{array}{l}\text { Deep water } \\
\text { telemetry }\end{array}$
\end{tabular}

Table 4. Example data rates for potential ranges of underwater EM.

radius is the achievable transmission range that can guarantee the optimized quality of signal reception, which is an improvement compared to the work in [13].

All measurement data will be sent to one destination node (i.e., the sink) on the shore or any other designated position. The sink will be equipped with a Global System for Mobile Communications (GSM) cellular radio, which allows all the collected data to be eventually transported to a central office for processing. This enables remote collection and processing of the data. The system will also gather data regarding the health of the sensor nodes which will also be accessible by the user, enabling failed nodes to be replaced as needed.

A small-scale wireless sensor network has been designed using a multihop approach. Six underwater sensor nodes are placed in two rows with a fixed topology, as shown in Fig. 3. The distance between every two adjacent nodes is set to $40 \mathrm{~m}$. In order to reduce the bottleneck transmission problem as investigated in [14], the sink node is placed in the center of the network and above the water surface. In this project, the short transmission range issue is not a major concern since the application and topology allow short links. The coverage of this network can generate a sufficient amount of data for the coastal research required in [19]. The fundamental purpose of this sensor network is to find the DoC and the movement of seabed sediment around this contour. It will only be necessary to monitor sea bed movement below the low tide line, and the sensors need to be positioned on either side of the closure con- 


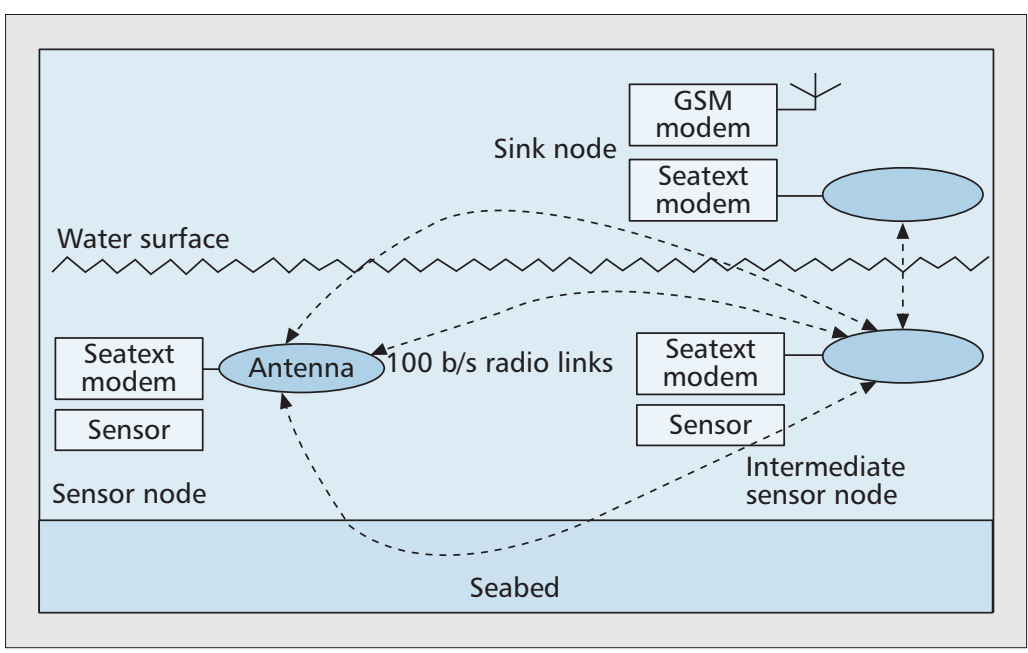

Figure 2. System schematics and multipath propagation.

tour. This will enable a temporal picture of sedimentary movement around this contour to be constructed.

As in most other wireless sensor networks, this network scenario follows multiple-source and single-destination traffic patterns. The data delivery is carried out in cycles. Within each cycle, after the data gathering period, all nodes stay in sleep mode until a scheduled transmission is initiated, and then the nodes wake up and are ready for communication. Once data transmission is complete, the nodes revert back to sleep mode.

\section{Control Protocol}

Networking protocol plays a vital role in saving power and providing consistent connections in underwater wireless communications, most of which have been designed for acoustic technologies [2]. Due to the unique characteristics of the system defined above, an efficient and effective control protocol needs to be carefully designed to meet the network specifications and requirements.

A few issues have to be addressed prior to the network protocol design: low data rate, halfduplex communication pattern, and the possible contention of resources in the burst transmission period. The network characteristics have led to the decision of using a variation of timedivision multiple access (TDMA) as the control protocol since it can resolve the problems addressed above. Each end sensor node will be allocated a time slot during which it can transmit the collected data to the sink. Initially, a static routing mechanism will be enabled for the data forwarding process, which is easy to implement in small-scale networks. The intermediate node involved in the predetermined route needs to wake up to relay the data. Within each time slot, the source node will follow this state transition: wake up $\rightarrow$ transmitting only $\rightarrow$ sleep; while intermediate nodes will follow the transition: wake up $\rightarrow$ receiving only $\rightarrow$ transmitting only $\rightarrow$ sleep. The above is, of course, a simplification that assumes no acknowledgment (ACK) packets are involved in the process of communications.
There are a few advantages of using TDMA as the control protocol. First, there will be no collision between any two transmissions during the burst traffic period. The end-to-end (ETE) data delivery process for each sensor node is completed within its own time slot. Second, it fits the half-duplex transceiver mechanism. The active sensor nodes are in either transmittingonly or receiving-only mode. Third, TDMA will be used as a cross-layer control protocol which is lightweight and efficient. Fourth, while one sensor node is transmitting data, all the uninvolved sensor nodes can stay in sleep mode in order to conserve battery life.

\section{Frame Design and Synchronization}

The system uses a flexible TDMA method of allocating time slots to each node in the network as a data collision avoidance mechanism. All the nodes in the network including the sink are allocated a time slot during which the transmitting node is able to send its data to the sink.

Data transmission uses Reed-Solomon (255, 233) forward error correction (FEC) [22], which sets 255 bytes as the maximum size of the data frame for each time slot. In order to enhance resource utilization and network efficiency, the data frame is set to the maximum size (i.e., 255 bytes). The complete frame size is set to 2128 bits including all physical layer information. Packets generated from different sensors will experience various ETE delays to reach the sink, including transmission delay $\Delta \mathrm{T}_{t x n}$ and propagation delay $\Delta \mathrm{T}_{\text {prop. }}$. When all the nodes are functioning properly, the maximum data delivery hop count is two. In case of node failure, the maximum hop count is three. For instance, when node 2 fails, node 4 has to go through node 5 and 3 to reach the sink, hence the maximum ETE delay $=3 \times\left(\Delta \mathrm{T}_{t x n}+\right.$ $\left.\Delta \mathrm{T}_{\text {prop }}\right)$. Should reliable transmission be enabled in the network, the slot length also needs to take ACK packets and retransmission into account. If three failures are allowed in the transmission, there can be four data frames $(2128 \times 4=8512$ bits $)$ and one ACK frame (set as 124 bits), which would take $8636 / 100 \approx 87 \mathrm{~s}$ to transmit. Including the guard period, overhead processing time, and propagation delay, the total time it takes to get a single frame across each link can be rounded up to $100 \mathrm{~s}$. The maximum number of hops is three, so the maximum slot size will be $3 \times 100=300 \mathrm{~s}$.

The frame types that are involved in the network communications include command frame, data frame, ACK frame, and synchronization frame. The key to the flexibility of this system is the ability to define virtually every parameter of the TDMA frame structure, which is achieved by using a command frame to program the TDMA scheduler. This approach facilitates optimal performance after network deployment. The details of these frame formats are not covered in this article, but can be found in [23].

In order for the system to function correctly, all nodes must have a synchronized clock or the slots will be incorrectly aligned, so a clock synchronization protocol will need to be employed to make sure that synchronization is maintained. Even with a good synchronization protocol, there 
will still be some drift of the clocks between the various nodes. A guard band is placed between all the time slots to allow for this problem.

Synchronization of the clocks will need to take place via a synchronization frame broadcast by the sink during its time slot. This will be around the time when a set of data gathering readings are transmitted. The suggested time synchronization scheme is loosely based on the Delay Measurement Time Synchronization (DMTS) method, which has been proven to be highly energy efficient and computationally lightweight since it requires only one time broadcast to synchronize all the nodes in a network [24]. Before the slot transmission commences, a one-way message will be propagated to all sensor nodes, which will compare the remote and local time stamps to obtain the line of best fit.

\section{RESULt ANALYSIS}

As described before, 255 bytes is the maximum size of the data frame for each time slot. The hypothesis was that the bandwidth cost for the overheads will compromise network efficiency; hence, the data payload field should be filled as much as possible. Simulations have been carried out for the network structure shown in Fig. 3, where the underwater transmission environment has been modeled based on the equations mentioned earlier. Results have shown that as long as the data payload field occupancy is over 50 percent, the network will deliver acceptable efficiency (i.e., more than 76 percent goodput).

Table 5 shows various total delays for different data packet sizes. Total delay includes ETE transmission delay and the waiting time prior to transmission. The waiting time consists of the duration for synchronization and command frames to cross the network, as well as the pending time for the allocated slot. The results indicate that the total networking time for one cycle transmission lasts roughly half an hour. The variation of frame size generates some fluctuation of network delay on the order of seconds; however, this jitter is not significant enough to impair the network performance, since the delay is measured in minutes in the context of this system.

Battery usage is an important performance parameter in the UWSN, which reflects the energy efficiency of the network protocol. Assuming that the maximum frame size is used, Fig. 4 shows the total power consumption of the sink, an end sensor node, and an intermediate sensor node for two TDMA transmission cycles. The results are based on the following measurements and estimation: the power supply current of the sensor node in its transmission state is 520 $\mathrm{mA}$; in its receiving state it is $210 \mathrm{~mA}$; and for the idle state it is $0.2 \mu \mathrm{A}$. The intermediate node consumes more power than the sink node, which was not expected. The explanation for this occurrence follows. Even though the sink node needs to stay awake for each time slot, the power for receiving data is much lower than the power needed for sending data. On the other hand, an intermediate node needs to be engaged in the transmission states three times in a TDMA cycle: once to transmit its own data, and twice to relay its neighbor's data. Hence, the overall power usage for the intermediate nodes will exceed

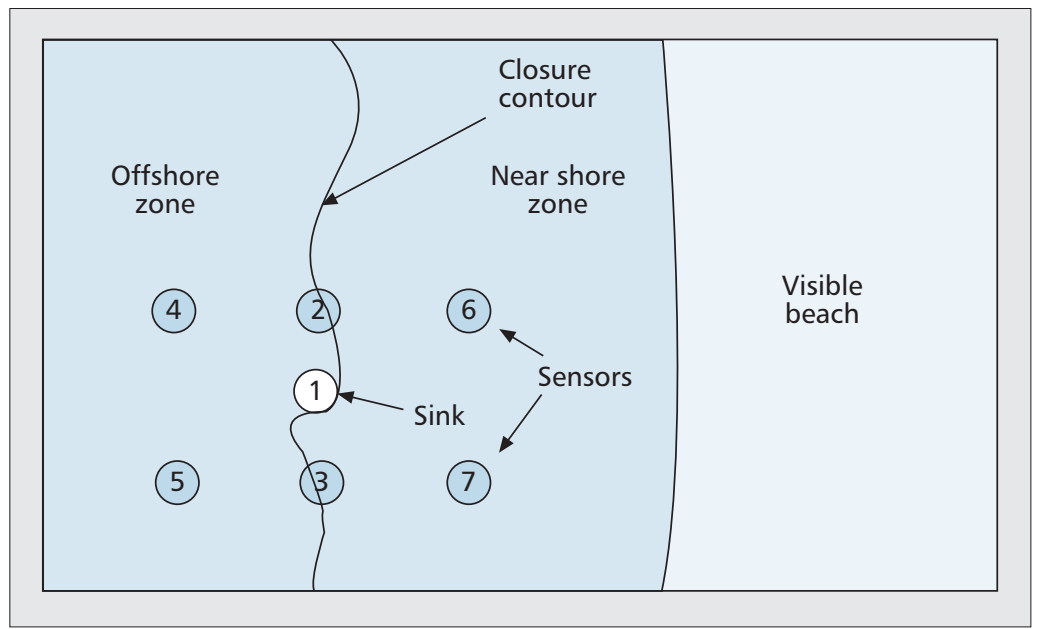

Figure 3. Network scenario.

\begin{tabular}{|lllllll|}
\hline Packet size & Node 4 & Node 2 & Node 6 & Node 5 & Node 3 & Node 7 \\
\hline 360 & 307.2 & 603.6 & 907.2 & 1207.2 & 1503.6 & 1807.2 \\
\hline 1100 & 322.0 & 611.0 & 922.0 & 1222.0 & 1511.0 & 1822.0 \\
\hline 2040 & 340.8 & 620.4 & 940.8 & 1240.8 & 1520.4 & 1840.8 \\
\hline
\end{tabular}

Table 5. Delay measurements (seconds).

that for the sink. Assuming that a compact and low-cost 18 Ah battery is employed for each node, and that two cycles of readings will be sent daily, the battery capacity will be able to maintain the network for approximately one year. This contributes to the plausibility of the deployment of a real system.

\section{Prospects and Challenges}

RF offers great potential for underwater communication that can contribute to fields such as environmental management, oceanography, and waste management. Since data transmission is unaffected by harsh weather conditions or ambient noise, the technology will operate in shallow and congested waters, thus enabling monitoring in complex environments such as ports and harbors, estuaries, and waterways.

Generally, lower radio frequencies are more desirable for underwater communications. In the past, extremely low frequency (ELF) submarine communication systems were believed to be the only successfully deployed subsea EM application $[25,26]$. The investigation of this article has shown that radio frequencies ranging from ultra low frequency (ULF) to medium frequency (MF) can be utilized in a variety of underwater environments.

However, several areas remain unexplored, and present unique challenges for underwater RF, including:

- As RF is susceptible to EMI, the effects of this interference is yet to be quantified. The available data rate is proportional to the bandwidth, and can also be affected by the signal-to-noise ratio. 


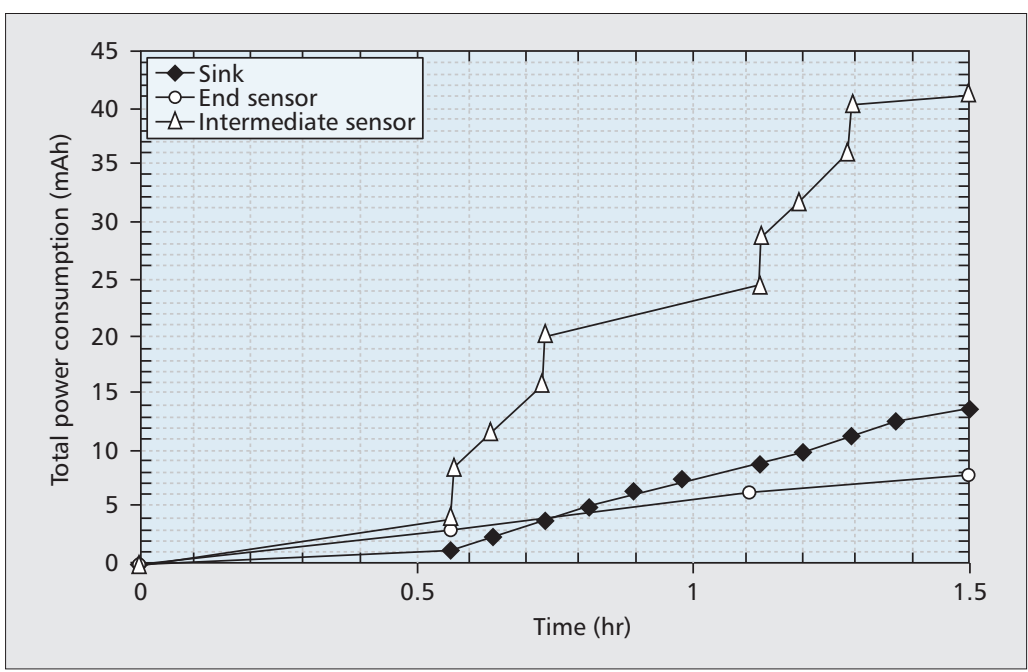

Figure 4. Total power consumption for two cycles.

- Even though RF presents advantages in shallow-water communication systems, there is still a range limitation in deep sea environments. The current applications in deep water are mostly based on close distance deployment.

- The antenna design for underwater RF systems has always been a concern. In the case study presented earlier, a half duplex antenna is a realistic solution for the scenario. Implementation of a duplex transceiver would not be impossible; however, there will be added cost and complexity to the system due to the size of the antenna.

- The data rates of underwater RF communication systems are frequency-dependent, whereas data rates of acoustic systems remain relatively constant. Given that the attenuation of an EM signal increases significantly with both frequency and distance, the choice of operating frequency, bit rate, and sensor density requires careful deliberation during the design phase.

When using RF technology in underwater scenarios, each solution has to uniquely balance parameters such as antenna design, transmit power, duty cycle, data bandwidth, antenna size, and local noise sources in order to achieve an optimized solution for the specific application.

\section{CONCLUSIONS}

Underwater EM communication has very few applications to date. Communications technology and operational requirements have radically changed since underwater electromagnetism was first evaluated. An overview and comparison of the three major underwater technologies (radio, acoustic, and optical) indicate the potential of underwater EM. Investigation has revealed that EM signaling, coupled with digital technology and signal compression techniques, has many advantages that make it suitable for niche underwater applications. In the case study presented, a small-scale underwater wireless sensor network is designed to monitor coastal erosions. EM communication is used in the physical layer because it presents distinct advantages compared to acoustic and optical communication in shallow water coastal environments. The analysis of this case study has shown that EM communication in underwater environments is both feasible and effective for a specific set of applications.

\section{APPENDIX A}

Based on [27], the refractive index of a medium can be presented using the following equation, where $\lambda$ is the wavelength in the medium, and $c_{0}$ is the speed of light.

$$
\frac{\lambda_{0}}{\lambda}=\frac{c_{0} k}{\omega}
$$

$\lambda_{0}$ is the wavelength in free space.

$$
\lambda_{0}=2 \pi c_{0} / \omega
$$

$k$ is the wave number of a good conductor [28], which is a complex number.

$$
k \cong e^{i \pi / 4} \sqrt{\mu_{0} \omega \sigma} .
$$

Hence, the real part of $k$ is

$$
R(k) \cong \sqrt{\mu_{0} \omega \sigma / 2} .
$$

$\omega$ is the angular frequency.

$$
\omega=2 \pi f
$$

If using Eqs. 6, 7, and 8 to substitute the $\lambda_{0}, k$, and $\omega$ in Eq. 5, the equation of wavelength can be derived as

$$
\lambda=2 \sqrt{\pi /\left(\mu_{0} f \sigma\right)} .
$$

Given the free space permeability $\mu_{0}=4 \pi \times 10^{-7}$ $\mathrm{H} / \mathrm{m}, \mathrm{Eq} .9$ can be further expressed as shown in Eq. 1.

$$
\lambda=1 / \sqrt{f \sigma \times 10^{-7}}
$$

Using $v=f \lambda$, the equation for propagation velocity is

$$
v=2 \sqrt{f \pi /\left(\mu_{0} \sigma\right)},
$$

or as shown in Eq. 2,

$$
v=\sqrt{f \times 10^{7} / \sigma} .
$$

Skin depth is given by [29]

$$
\delta_{\text {skin }}=1 / \sqrt{f \pi \sigma \mu_{0}} .
$$

Using $\mu_{0}=4 \pi \times 10^{-7} \mathrm{H} / \mathrm{m}$, the skin depth equation can be shown as in Eq. 3 .

$$
\delta_{\text {skin }}=1 /\left(2 \pi \sqrt{f \sigma \times 10^{-7}}\right)
$$

\section{REFERENCES}

[1] R. K. Moore, "Radio Communication in the Sea," IEEE Spectrum, vol. 4, Nov. 1967, pp. 42-51.

[2] D. Pompili and I. F. Akyildiz, "Overview of Networking Protocols for Underwater Wireless Communications," IEEE Commun. Mag., Jan. 2009, pp. 97-102. 
[3] M. Chitre, S. Shahabudeen, and M. Stojanovic, "Underwater Acoustic Communications and Networking: Recent Advances and Future Challenges," Marine Tech. Soc. J., vol. 42, 2008, pp. 103-16.

[4] R. Otnes et al., "A Roadmap to Ubiquitous Underwater Acoustic Communications and Networking," Proc. 3rd Int'l. Conf. Underwater Acoustic Measurements: Tech. \& Results, June 2009.

[5] E. M. Sozer, M. Stojanovic, and J. G. Proakis, "Underwater Acoustic Networks," IEEE J. Oceanic Eng., vol. 25, no. 1, Jan. 2000, pp. 72-83.

[6] J. Partana, J. Kurosea, and B. N. Levinea, "A Survey of Practical Issues in Underwater Networks," ACM Mobile Comp. Commun. Rev., vol. 11, 2007, pp. 23-33.

[7] H. Riksfjord, O. T. Haug, and J. M. Hovem, "Underwater Acoustic Networks - Survey on Communication Challenges with Transmission Simulations," Proc. 3rd IEEE SENSORCOMM, June 2009, pp. 300-5.

[8] W. Au, P. Nachtigall, and J. Pawloski, "Acoustic Effects of the ATOC Signal $(75 \mathrm{~Hz}, 195 \mathrm{~dB})$ on Dolphins and Whales," J. Acoustical Soc. Amer., vol. 101, May 1977, pp. 2973-77.

[9] A. Reza and J. Harms, "Robust Grid-Based Deployment Schemes for Underwater Optical Sensor Networks," Proc. 34th IEEE Conf. LCN, Oct. 2009, pp. 641-48.

[10] D. Anguita, D. Brizzolara, and G. Parodi, "Optical Communication for Underwater Wireless Sensor Networks: A VHDL-Implementation of a Physical Layer 802.15.4 Compatible," Proc. IEEE Oceans Europe, May 2009.

[11] S. Arnon and D. Kedar, "Non-Line-Of-Sight Underwater Optical Wireless Communication Network," J. Optical Soc. Amer., vol. 26, Mar. 2009, pp. 530-39.

[12] J. H. Smart, "Underwater Optical Communication Systems," Proc. IEEE MILCOM, vol. 2, Oct. 2005, pp. $1140-46$.

[13] U. M. Cella, R. Johnstone, and N. Shuley, "Electromagnetic Wave Wireless Communication in Shallow Water Coastal Environment: Theoretical Analysis and Experimental Results," Proc. 4th ACM Int'l. Wksp. UnderWater Net., Nov. 2009.

[14] X. Che et al. "A Static Multi-Hop Underwater Wireless Sensor Network Using RF Electromagnetic Communications," Proc. 2nd IEEE Int'l. Wksp. Specialized Ad Hoc Net. Sys., June 2009, pp. 460-63.

[15] P. Smith, "Measurement and Analysis of Transmit Antenna Configurations for Underwater RF Communications," Proc. 3rd SEAS DTC Tech. Conf., 2008.

[16] R. King, "Lateral Electromagnetic Waves from a Horizontal Antenna for Remote Sensing in the Ocean," IEEE Trans. Antennas Propagation, vol. 37, Oct. 1989, pp. 1250-55.

[17] M. S. Roden, Analog and Digital Communication System, 3rd ed., Prentice Hall, 1991, p. 442.

[18] M. Rhodes, B. Hyland, and D. Wolfe, "Underwater Navigation," U.K. Patent 2462543, Feb. 2010.

[19] M. R. Phillips and A. T. Williams, "Depth of Closure and Shoreline Indicators - Empirical Formulae for Beach Management," J. Coastal Research, vol. 23 2007, pp. 487-500.

[20] I. Crowther, "Through-Water and Through-Ground Radio Modem: Seatext," KM Seatext E\&I, Wireless Fibre Systems, May 2009.

[21] M. Rhodes, B. Hyland, and D. Wolfe, "Underwater Communications System," U.S. Patent 134331, Dec. 2006.

[22] A. Rashid et al., "A Low Complexity, High Speed, Regular and Flexible Reed Solomon Decoder for Wireless Communication," IEEE Design \& Diagnostics Electronic Circuits Sys., 2006, pp. 31-36.

[23] X. Che et al.. "Frame Design for a Prototype Underwater RF Electromagnetic Communication Sensor System," ASTEC Internal Publications; http://www. fishnetz.co.uk/Publications.html.

[24] I. Rhee et al., "Clock Synchronization in Wireless Sensor Networks: An Overview," Sensors, vol. 9, 2009, pp. 56-85.

[25] U.S. Navy, "Extremely Low Frequency Transmitter Site: Clam Lake, Wisconsin," U.S. Navy Facts File, June 2001.

[26] T. B. Sanford and R. H. Tyler, "Nearshore Navigation and Communication Based on Deliberate EM Signals, Numerical, and Observational Studies of Coastal Ocean Electrodynamics," Univ. WA, Applied Physics Lab., Apr. 2005.
[27] J. D. Jackson, Classical Electrodynamics, Wiley, 1998.

[28] R. Fitzpatrick, "Advanced Classical Electromagnetism," Univ. TX, 1996.

[29] D. Griffiths, Introduction to Electrodynamics, Pearson Education, 2003.

\section{BIOGRAPHIES}

XIANHUI CHE [M] (xianhui.che@smu.ac.uk) obtained her B.Eng. in computer information and network systems from the North China Electric Power University, Beijing, in 2001 and her M.Sc. in telecommunication and information systems from the University of Essex in 2002; the same year, she was granted a full Ph.D. scholarship. She gained her doctorate degree in electronic systems engineering in 2006. She is now working as a senior lecturer in the School of Applied Computing at Swansea Metropolitan University, United Kingdom. She has been involved in several projects throughout her research pathway and delivered various publications. Her research interests include TCP/IP data networking, network protocols, network modeling and simulations, optical and wireless networking, and game networks. She has reviewed papers for several journals.

IAN WeLLS [M'90, SM'07] (ian.wells@smu.ac.uk) obtained a B.Sc. in physics from Manchester University, United Kingdom, in 1982, an M.Sc. in microelectronics and digital signal processing from the University of Westminster London, United Kingdom, in 1986, and a Ph.D. in signal processing architectures for speech recognition from the University of the West of England, Bristol, in 1996. He is currently head of the School of Applied Computing at Swansea Metropolitan University, where he has worked since 1996. He was previously the research director of Technium Digital, University of Wales, Swansea. His current research interests include ultrasonic nondestructive testing, medical signal processing, communications systems, and computer networks. He is also a Fellow the Institution of Engineering and Technology (FIET), a Fellow of the British Computer Society (FBCS), and a Chartered Engineer (C.Eng.).

GORDON A. DICKERS [M] (gordon.dickers@smu.ac.uk) obtained a B.Sc. in electronic and electrical engineering from Swansea University in 1985 and an M.Sc. in computing with distinction from Cardiff University, United Kingdom, in 2001. He is currently a senior lecturer in the School of Applied Computing at Swansea Metropolitan University, where he has worked since 2001 . He also worked as a software engineer and electronic design engineer in Lucas Aerospace, United Kingdom, after completing his first degree. He has taught in further and higher education institutions in the United Kingdom since 1991. His current research interests include medical signal processing, Al, computer graphics and vision, and computer networks. He is also a member of the IEEE Computer Society and the British Computer Society (MBCS).

PAULKEAR (paul.kear@smu.ac.uk) is currently a senior lecturer in the School of Applied Computing at Swansea Metropolitan University and is currently completing his Ph.D. He gained both his first class undergraduate (1998) and postgraduate degrees (2006) with distinction from Swansea Metropolitan University. His main research interest is in underwater ad hoc wireless sensor networks. He started his career in the petrochemical sector and has 25 years of embedded system, control system, transducer, and network design expertise. He also has system design experience in the pharmaceutical sector. He is a paper reviewer for IEEE ICC and IEEE GLOBECOM.

XIAOCHUN GONG (xiaochun.gong@smu.ac.uk) joined the network research team of the School of Applied Computing in Faculty of Applied Design and Engineering in the beginning of 2009. He is a senior research associate at Swansea Metropolitan University. He obtained his Ph.D. and first degrees from universities in China. His research interests include $\mathrm{C}++$ and $\mathrm{C \#}$ programming and electronic engineering. His major contribution to the ASTEC project was system implementation with embedded programming. 